\title{
Independent Representation of Parts and the Relations Between Them: Evidence From Integrative Agnosia
}

\author{
Marlene Behrmann \\ Carnegie Mellon University \\ Morris Moscovitch \\ University of Toronto
}

\author{
Mary A. Peterson \\ University of Arizona
}

Satoru Suzuki

Northwestern University

\begin{abstract}
Whether objects are represented as a collection of parts whose relations are coded independently remains a topic of ongoing discussion among theorists in the domain of shape perception. S.M., an individual with integrative agnosia, and neurologically intact ("normal") individuals learned initially to identify 4 target objects constructed of 2 simple volumetric parts. At test, the targets were mixed with distractors, some of which could be discriminated from the targets on the basis of a mismatching part, whereas the rest could be discriminated only on the basis of the altered spatial arrangements of parts. S.M. learned to identify the target objects, although at a rate slower than that of the normal participants. At test, he correctly rejected distractors on the basis of mismatching parts but was profoundly impaired at rejecting distractors made of the same local components but with mismatching spatial arrangements. These results suggest that encoding the spatial arrangements of parts of an object requires a mechanism that is different from that required for encoding the shape of individual parts, with the former selectively compromised in integrative agnosia.
\end{abstract}

Keywords: agnosia, object perception, neuropsychology, vision, visual representations

Despite decades of research on shape perception, there still remains much controversy concerning the codes underlying object representation. One theoretical approach posits that a threedimensional object is represented explicitly by a small number of volumetric primitives combined with their spatial relationships (Biederman, 1987; Hoffman \& Richards, 1984; Hummel, 1994; Marr \& Nishihara, 1978; Palmer, 1977). Although earlier versions of this structural description approach were challenged on empirical, theoretical, and computational grounds (Edelman, 1997), more recent versions address some of the criticisms by allowing for both preserved metric information about size and shape, which permits more robust within-category discrimination (Biederman, Subramaniam, Bar, Kalocsai, \& Fiser, 1999), and some consideration of the role of experience in object perception (Hummel \& Stankiewicz, 1996, 1998), which permits differential performance on different classes of objects as a function of expertise (Palmeri $\&$ Gauthier, 2004). In spite of these advances, many aspects of the

Marlene Behrmann, Department of Psychology, Carnegie Mellon University; Mary A. Peterson, Department of Psychology, University of Arizona; Morris Moscovitch, University of Toronto, Toronto, Ontario, Canada; Satoru Suzuki, Department of Psychology, Northwestern University.

This work was supported by National Institutes of Health (NIH) Grant MH 54246 to Marlene Behrmann, National Science Foundation (NSF) Grants BCS 9906063 and 04256520 to Mary A. Peterson, NSF Grant SBR-9817643 and NIH Grant EY 14110 to Satoru Suzuki, and a grant from the Canadian Institute of Health Research to Morris Moscovitch.

Correspondence concerning this article should be addressed to Marlene Behrmann, Department of Psychology, Carnegie Mellon University, Pittsburgh, PA 15213-3890. E-mail: behrmann@cmu.edu structural description approach remain underspecified; for example, despite some progress on the issue (Hummel, 2001), it is still not exactly clear how shapes are segmented into a collection of parts in the first place or how parts are assembled into configurations (Saiki \& Hummel, 1998a, 1998b). Moreover, the extent to which the derivation of parts is really independent of the coding of the spatial relationships is still a matter of ongoing debate. The focus of this article is on this last point, with specific emphasis on the potential separability of part and relation processing in object perception, as predicted by structural description accounts (Hummel \& Biederman, 1992), and it is addressed by examining how objects are represented by a person with a neurological disorder that affects object recognition.

\section{PARTS AND THEIR RELATIONS: NEUROPSYCHOLOGICAL INVESTIGATIONS}

Whether there are separable processes for recognizing objects and their parts has been the focus of some previous investigations, and some data to support their independence have already been obtained. For example, some developmental studies report a shift in representations for visual recognition from one that is more partbased to one that is more whole-based as children mature. In one such set of investigations, Davidoff and Roberson (2002), in a matching task using images of either intact whole animals or subparts of different animals, showed that children aged 10 and 11 years exhibited fair knowledge of animal parts but performed rather poorly on their ability to match the whole animals when the overall shape was transformed (e.g., by changing size). Remarkably, the development of the adult ability to recognize the whole 
shape of the animal was only achieved in their sample at 15 or 16 years of age. This late development of more holistic shape representations is also evident in children up to 14 years of age when required to learn to recognize novel three-dimensional artificial shapes (Rentschler, Juttner, Osman, Muller, \& Caelli, 2004). The reliance on part-based processing is also occasionally evident for adults under certain conditions: For example, when exposed to three-dimensional computer-generated shapes (resembling wire shapes) that differed in rotation or number of parts or in the curvature, length, or angle of join of the component parts, adult observers appeared to rely on a viewpoint-invariant parts-based process to differentiate between these unknown and somewhat impoverished shapes (Foster \& Gilson, 2002).

A different approach to understanding whether objects are indeed collections of parts whose relations are coded separately is to examine the performance of individuals who appear to have access to components or parts of shapes but seem unable to bind them into a unified whole. Such individuals suffer from integrative agnosia (IA), the term coined specifically to denote a subtype of visual agnosia in which individuals suffer from poor perceptual integration of local parts into higher order shapes (Behrmann \& Kimchi, 2003; Butter \& Trobe, 1994; Grailet, Seron, Bruyer, Coyette, \& Frederix, 1990; Kartsounis \& Warrington, 1991; Piccini, LauroGrotta, Michela Del Viva, \& Burr, 2003; Riddoch \& Humphreys, 1987; Thaiss \& de-Bleser, 1992; Wapner, Judd, \& Gardner, 1978). Although object identification may succeed at times, failures abound, perhaps as a result of inappropriate or excessive segmentation, with errors consisting mostly of guesses based on a local part; for example, Patient R.N. called a harmonica a "computer" (presumably perceiving the small openings arranged in orderly rows as keys on a keyboard). The recognition deficit applies equally to the recognition of two- and three-dimensional stimuli and to black-and-white and chromatic displays, although, in almost all cases, the presence of depth, color, and surface cues may be of some assistance to the patients in segmenting or binding together parts of the display (Chainay \& Humphreys, 2001; Farah, 1990, 2004; Humphrey, Goodale, Jakobson, \& Servos, 1994; Jankowiak, Kinsbourne, Shalev, \& Bachman, 1992; Ricci, Vaishnavi, \& Chatterjee, 1999). As with other forms of agnosia, the recognition deficit cannot be attributed to a problem in labeling the stimulus per se, nor to a loss of semantics; presented with the same object in a different modality, either haptically or auditorily, these individuals have no problem in naming or providing detailed and rich descriptions of it (Farah, 1990, 2004; Humphreys \& Riddoch, 2001; Ratcliff \& Newcombe, 1982).

Evidence to support the idea that lower level visual representations are intact but that their integration to higher order representations is impaired comes from several sources (Humphreys \& Riddoch, 2006; Piccini et al., 2003). Several individuals with IA perform more poorly when displays are presented briefly (presumably because time is required to assemble the component parts) and when object depictions are complex rather than simple (again, presumably taxing the integration system; Humphreys, 1999; Humphreys \& Riddoch, 2006; Humphreys et al., 1994). Drawings of overlapping objects are particularly poorly recognized, ostensibly because parts are less obviously discernible (e.g., Behrmann, Moscovitch, \& Winocur, 1994). In addition, discriminating real from pseudo-objects (also sometimes called nonobjects), whose parts are legitimate, is disproportionately difficult for many of these individuals.

Many patients with IA also have trouble perceiving global compound shapes composed of local letters (Behrmann \& Kimchi, 2003; Ricci et al., 1999; but see Humphreys, Riddoch, \& Quinlan, 1985; Riddoch \& Humphreys, 1987) and are impaired in conjoining line segments into simple, known shapes. For instance, Patients S.M. and R.N. were impaired at deriving a configural whole from four nonabutting line segments, arranged to form the shape of a diamond (Behrmann \& Kimchi, 2003). In addition, some patients with IA have difficulty perceiving collinearity and subjective contours and in interpolating contours (Kartsounis \& Warrington, 1991; Piccini et al., 2003), although others, such as H.J.A., have no difficulty linking collinear local features into edges except when occlusion is present in the image (Giersch, Humphreys, Boucart, \& Kovács, 2000). These deficits may reflect difficulties constructing whole edges from segments and further attest to the challenges posed in assembling global wholes from more local elements. One particularly clear example that illustrates this problem in integrating basic, local visual elements into perceptual wholes is Patient H.J.A.'s performance on a visual search task, requiring the detection of an inverted $T$ among distractors (Humphreys, 1999; Humphreys \& Riddoch, 1987; Humphreys et al., 1994; Humphreys, Riddoch, Quinlan, Price, \& Donnelly, 1992): H.J.A. performed as well as controls when the distractors were $T \mathrm{~s}$ in varying orientations (e.g., $90^{\circ}$ rotated left, $90^{\circ}$ rotated right, and upright) but performed significantly more poorly than the controls in the same task when the distractors were homogeneous. In the latter case, normal individuals can typically group the homogeneous distractors together, and the target can easily be segmented with the result that target detection is fast and independent of the number of distractors in the image. Given that H.J.A. cannot group the elements in parallel, he did not benefit from the distractor homogeneity and, as was true in the heterogeneous distractor trials, his response times (RTs) reflected the number of distractors in the display.

\section{IA AS FAILURE TO BIND LOCAL PARTS?}

Although the impaired ability to integrate parts into a global whole is consistent with this set of IA symptoms, two interpretative problems arise. The first is that the problem may not be one of integration per se and may simply be a consequence of a deficit in shape perception, a deficit that may be evident when the task is difficult. In this alternative view, the label "integrative agnosia" is a misnomer-because parts are simpler to identify than wholes, part identification but not whole shape identification may be preserved. Thus, it is not the integration or relations between parts per se that is affected, but rather shape processing is limited and only simple shapes are perceived. Other variants of this view are also possible: For example, if there is a fixed probability that a shape (part or whole) will be identified, then there will be more successes with parts than with wholes by virtue of the parts being more numerous. Another example is that the failure to report the whole may arise from an attentional deficit; some studies have suggested that the bottleneck arises because of a limited attentional window, with the result that patients only perceive small portions of the input rather than the entire stimulus (Coslett, Stark, Rajaram, \& Saffran, 1996; Thaiss \& de-Bleser, 1992). In this situation, 
report of parts is also more successful than report of the whole. A final variant is that integration of parts may simply be more difficult than perception of the parts (e.g., integration may require additional feature processing or attentional allocation) with the result that, following any form of damage, it is the former rather than the latter that is disproportionately affected.

The second interpretive problem concerns what constitutes a part for object perception and recognition. The definition of a part is extremely elusive, and one shape's part is another shape's whole: A wheel on a bicycle is certainly a part, but it is the whole for the spokes. Are patients with IA impaired at all forms of integration needed to form wholes at various levels, or are they more impaired at some forms of integration than others? For example, can they represent objects as a collection of volumetric parts but be impaired at representing their spatial arrangement? If this latter case holds, then this would provide evidence for the separable coding of parts and their relations and would shed light on the mechanisms underlying normal object perception.

\section{THE PRESENT STUDY}

To address the separability of parts and their relations in the present study, we trained S.M., an individual with IA, to identify four novel objects constructed from eight volumetric component parts (see Figure 1A). Several theories have argued that the parts of many objects can be modeled as volumetric components, such as generalized cylinders (Biederman, 1987; Binford, 1971; Marr \& Nishihara, 1978). The parts used to construct the objects in the present study are roughly akin to geons, which are simple threedimensional volumetric shapes made of local features, and it is from these geons that object representations are built (Biederman, 1987). Objects themselves can be described as a set of geons arranged in particular spatial relationships. The question, then, is whether patients with IA are equally impaired at integrating local features into geons and geons into objects or whether the deficit is more pronounced for one type of integration than the other.

S.M. and age-matched control participants were trained to identify the four novel objects shown in Figure 1A. Later, at test, we showed the participants the original four targets, presented either from the studied viewpoint or from a new vantage point, and asked them to discriminate instances of the targets from two types of distractors: part-changed (PC) and relation-changed (RC) distractors (see Figures 1B and 1C). PC distractors were constructed by replacing a part of each target with a part from another target. Because PC distractors could be rejected on the basis of a mismatching part, performance on these distractors could be used to assess the participants' ability to identify individual geons in the context of two-geon objects. RC distractors were constructed by altering the spatial arrangement of the original two geons of each target. Because RC distractors could be distinguished from the targets only on the basis of differences in the spatial arrangement of the geon parts, disproportionate difficulty in rejecting RC distractors relative to the $\mathrm{PC}$ distractors would indicate a selective impairment in the processing of the spatial arrangements of geon parts.

S.M. showed the expected pattern, indicating a selective impairment in processing the spatial arrangements of geon parts. We conducted additional investigations to address the essential question of whether S.M.'s reliance on local parts for identification is merely a limitation in shape processing or, alternatively, whether the local parts are indeed available but their spatial integration is impaired. Our results show that the latter is the case, thereby supporting the claim that parts may be accessed during object perception, independent of their spatial relations.

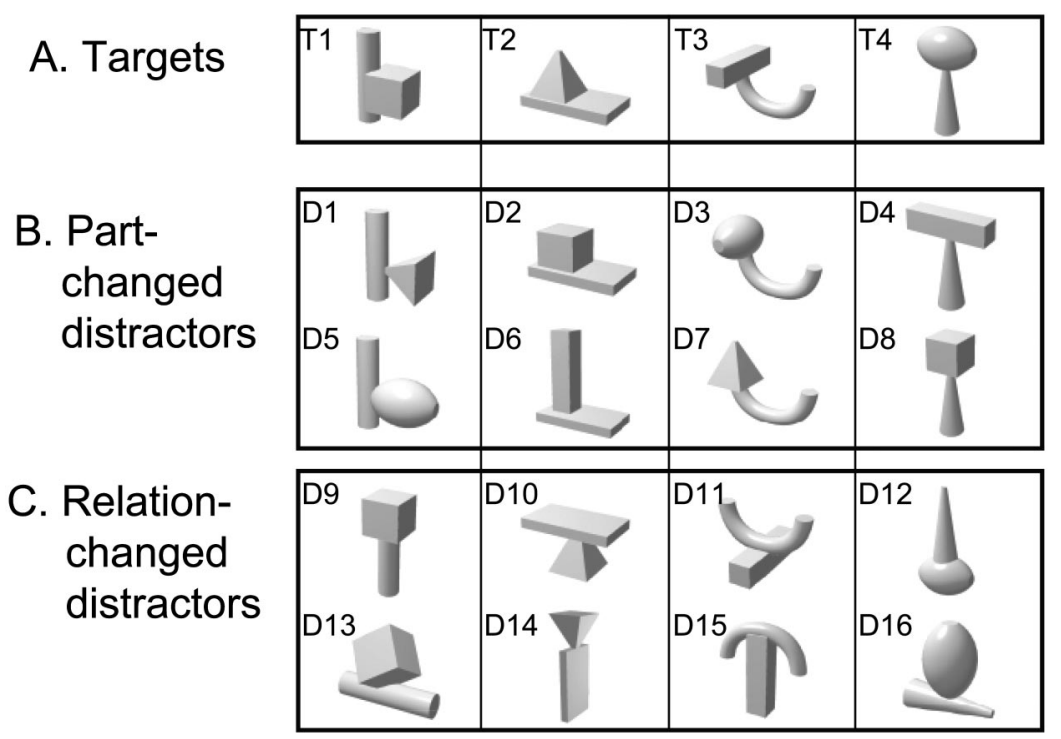

Figure 1. A: The 4 two-geon targets (labeled T1-T4) that the participants learned to identify. B: The eight part-changed distractors (D1-D8) that are generated by replacing one of each target's parts with a part from a different target. C: The eight relation-changed distractors (D9-D16), which are generated by scrambling the spatial arrangement of the two parts. The frequency of occurrence of each part was identical for the two types of distractors. 


\section{METHOD \\ Participants}

Participants included 1 patient with IA, S.M., and 42 undergraduates from the University of Arizona, who served as control participants. All participants had normal or corrected-to-normal acuity and were tested individually. S.M.'s case study follows.

At the time of this testing in 1997, S.M. was a 21-year-old, right-handed, English-speaking man, who had sustained damage primarily to the right inferior temporal lobe in a motor vehicle accident in 1994, although a deep shearing injury in the corpus callosum and left basal ganglia were also noted on a previous scan (see Figure 2 for a recent structural magnetic resonance imaging scan; note that for ease of depiction, the right of the brain is on the right of the figure).

S.M. recovered well from his injury, and his residual behavioral impairment is in visual object and face processing. S.M. has participated in many previous studies, and the reader is referred to those for further details (Behrmann \& Kimchi, 2003; Behrmann, Marotta, Gauthier, Tarr, \& McKeeff, 2005; Gauthier, Behrmann, \& Tarr, 1999, 2004; Humphreys, Avidan, \& Behrmann, in press). In brief, neuro-ophthalmological examination confirmed no visual sensory deficit in S.M.: He has visual acuity of 20-20 bilaterally, and his eyes are unremarkable for pathology of any form. S.M. showed no evidence of hemispatial neglect on a standard bedside battery (Black, Vu, Martin, \& Szalai, 1990). Table 1 summarizes his performance across a range of neuropsychological tests. He performed within the normal range on subtests that assess basic aspects of visual perception, including localizing a dot in relation to a frame, form and line orientation discrimination, dot counting, and simple figure-ground segregation. He was also able to match objects and to copy even complex drawings reasonably well, presumably because the local components can be encoded serially.

S.M., however, was more impaired on tests of overlapping shapes than tests with single items and was generally impaired on all tasks that require visual access of object knowledge. His recognition and discrimination of common objects is notably poor: He performs significantly more slowly and with errors, relative to control participants, in discriminating between common objects and even between novel objects, such as greebles or snowflakes (Gauthier et al., 1999). He was able, with time and effort, to learn to discriminate between individual greebles, but this was done at
Table 1

Neuropsychological Test Scores for S.M.

\begin{tabular}{|c|c|}
\hline Test & Score \\
\hline \multicolumn{2}{|l|}{ Lower level visual processing } \\
\hline Visual Object and Space Perception Battery & $\begin{array}{l}\text { Normal range on all } \\
\text { subtests }\end{array}$ \\
\hline Benton Visual Form Discrimination ${ }^{\mathrm{b}}$ & Low average \\
\hline Benton Line Orientation $^{\mathrm{b}}$ & Low average \\
\hline Efron Shape Matching Task ${ }^{\mathrm{c}}$ & $24 / 25$ \\
\hline \multicolumn{2}{|l|}{ Birmingham Object Recognition Battery ${ }^{\mathrm{d}}$} \\
\hline Line Length (Test 2) & Normal \\
\hline Size (Test 3) & Normal \\
\hline Orientation (Test 4) & Normal \\
\hline Gap Position (Test 5) & Normal \\
\hline Overlapping Shapes (Test 6) & Impaired \\
\hline Minimal Feature Match (Test 7) & Normal \\
\hline Foreshortened Views (Test 8) & Normal \\
\hline Object Decision (Test 10 ) & Impaired \\
\hline \multicolumn{2}{|l|}{ Object recognition } \\
\hline Boston Naming Test ${ }^{\mathrm{e}}$ & $35 / 60$ \\
\hline Snodgrass and Vanderwart Pictures ${ }^{\mathrm{f}}$ & $172 / 259(66 \%)$ \\
\hline \multicolumn{2}{|l|}{ Face processing } \\
\hline Benton Facial Recognition Test ${ }^{\mathrm{g}}$ & 36/54; impaired \\
\hline Reading & $\begin{array}{l}\text { Slow but accurate; } \\
104 \mathrm{~ms} / \text { word }\end{array}$ \\
\hline Copying line drawings/images & Slow but accurate \\
\hline
\end{tabular}

${ }^{\mathrm{a}}$ Warrington \& James (1991). $\quad{ }^{\mathrm{b}}$ Benton et al. (1983). $\quad{ }^{\mathrm{c}}$ Efron (1968).

${ }^{\mathrm{d}}$ Riddoch \& Humphreys (1993). ${ }^{\mathrm{e}}$ Goodglass et al. (1983). ${ }^{\mathrm{f}}$ Snodgrass \& Vanderwart (1980). ${ }^{\mathrm{g}}$ Benton et al. (1978).

some cost to his already poor face identification (Behrmann et al., 2005). His identification scores on the Boston Naming Test (Kaplan, Goodglass, \& Weintraub, 1976) and the Snodgrass and Vanderwart picture set were abnormally low, with better performance on nonliving than living items $(122$ correct out of 165 vs. 50 correct out of 94$): \chi^{2}(1, N=259)=10.6$, $p<.001$. S.M.'s errors were predominantly misinterpretations of visual attributes, such as calling an acorn a "coconut" and a harmonica a

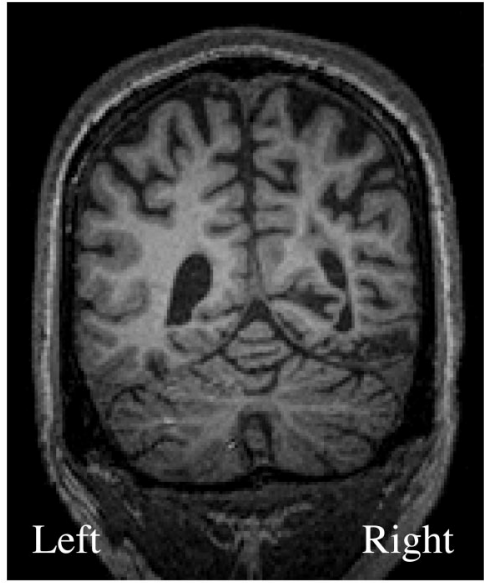

(a)

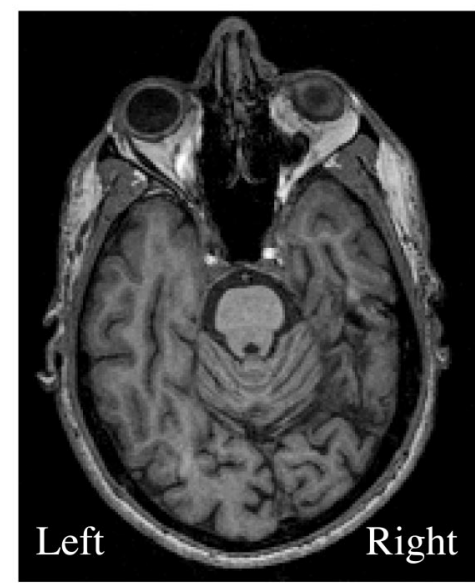

(b)

Figure 2. Recent (2005) structural magnetic resonance imaging scan for Patient S.M. showing coronal (a) and axial (b) sections through the lesion site in right inferior temporal lobe. 
"cashier's register." When he failed to recognize an item, he did not appear to possess any semantic or action information about the item. However, he was able to provide appropriate definitions to the auditory labels for those pictures he named incorrectly when visually presented. In addition, his object recognition from tactile input (without vision) of 34 objects was normal. Although S.M.'s performance on identifying the global and the local letters of Navon compound shapes (e.g., a large $\mathrm{H}$ made of small $\mathrm{Hs}$ or of small $\mathrm{Ss}$ ) was not significantly abnormal, his ability to derive a configural whole from local components is compromised when the number of local components is few (and thus the spatial relations between the parts must be emphasized strongly) and when there is insufficient time to bind together the local parts (Behrmann \& Kimchi, 2003).

It is not surprising that S.M.'s face processing is also markedly impaired. His performance (score of 36) on the Benton Facial Recognition Test (Benton, Sivan, Hamsher, Varney, \& Spreen, 1983) was in the impaired range, and he was unable to recognize any of the pictures of famous faces, including Bill Clinton (the president of the United States at the time), Sylvester Stallone (an actor), Elvis Presley (a singer), and Steve Martin (a comedian-actor). S.M.'s reading performance is accurate, although extremely slow, and he shows the letter-by-letter pattern with a monotonic increase in reading time as a function of word length (104 ms per additional letter).

S.M. thus appears to have normal visual acuity, normal ability to perceive basic visual features, and normal ability to match common objects across different views. He does, however, have difficulty parsing overlapping patterns, identifying common objects (especially, but not limited to, living items and faces), and processing whole words. All of these findings are consistent with the diagnosis of IA.

\section{Stimuli}

The stimuli were gray-scale renditions of shaded three-dimensional objects (approximately $4.5^{\circ} \times 4.5^{\circ}$ ) presented on a white background. Figure 1A shows the four targets that the participants were asked to learn to identify. Each target consisted of two distinct parts (geons), one of which was considered the supporting geon. The target was shown in a nonaccidental view (for both geon parts). No two targets shared a part.
For each target, five additional views were created, two views through rotations in the picture plane and three views through rotations in depth. As shown in Figures 3B and 3C, the two picture-plane rotations were by $90^{\circ}$ and $180^{\circ}$ clockwise and the depth rotations were $-30^{\circ}, 30^{\circ}$, and $60^{\circ}$ counterclockwise around the vertical axis, where, for convenience of notation, positive angles correspond to rightward rotations. In addition to these views of the target, two PC distractors and two RC distractors were created from each target, resulting in eight distractors of each type (as in Figures $1 \mathrm{~B}$ and $1 \mathrm{C})$. For the PC distractors, only nonsupporting parts were exchanged across the four targets so as to preserve the basic global structure of each target in the corresponding distractors. Furthermore, each part appeared equally frequently (twice) across the eight PC distractors. For the RC distractors, the geons were retained but their spatial arrangements were altered (e.g., side-to-side attachment was changed to side-to-end attachment or end-to-end attachment). Hence, although the same two components were preserved, the global structure of the RC shapes was substantially different from that of the targets from which they were created.

\section{Procedure}

All experimental trials were controlled by a Macintosh IIcx computer using the experimental software, Vision Shell (Micro ML inc., Montreal, Quebec, Canada; www.visionshell.com). The procedure consisted of two parts: (a) a study phase (with a final assessment of learning) and (b) a test phase comprising two blocks of test trials. These two parts are described below. S.M. participated in two complete sessions. His performance did not differ across the two sessions; therefore, his results are collapsed and calculated over twice as many trials as the results for individual control participants.

\section{Study Phase}

The participants viewed a display like that shown in Figure 1A arrayed across the top of a computer monitor. They were asked to learn to identify the targets by labeling them as Target 1 , Target 2 , Target 3 , or Target 4 .

\section{A. Targets}

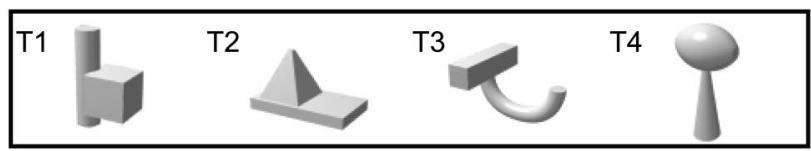

\section{B. Picture-plane rotation}

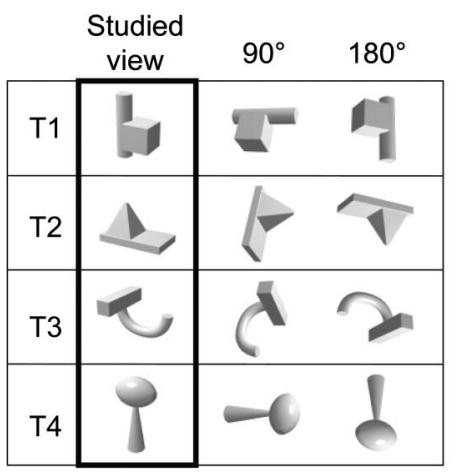

\section{Depth rotation}

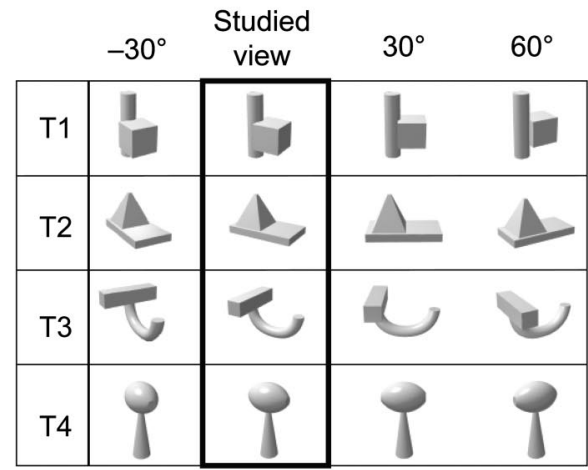

Figure 3. Targets in the studied view and from new orientations. A: The four targets shown for convenience. B: The two picture-plane rotated views of the four targets. The rotations were clockwise by $90^{\circ}$ and $180^{\circ}$ relative to the studied views. C: The three depth-rotated views (about the vertical axis) of the four targets. The rotations were counterclockwise about the vertical axis by $-30^{\circ}, 30^{\circ}$, and $60^{\circ}$, relative to the studied views. 
Half of the normal participants $(n=21)$, as well as S.M., viewed the display exactly as shown in Figure 1A. The left-to-right order of the targets (but not the numbers) was reversed for the remaining participants $(n=21)$. Left-right target order did not interact with any of the obtained effects. Participants were instructed that they would be asked later to identify the targets from different viewpoints. They were also told that they would be asked to discriminate the targets from distractors that would appear very similar to the targets. It is important to note that this instruction was given to prevent the participants from using a strategy of remembering each target in terms of only one of the two parts. Second, we emphasized to the participants that the two parts in each target were firmly fixed and could not slide around.

Following these instructions (given verbally while the participants were free to view the four targets that were arrayed across the top screen), the control participants were given 1 min to study the target display. S.M. was asked to tell the experimenter when he was ready to go on; he required just over $3 \mathrm{~min}$ in the first session and $1.5 \mathrm{~min}$ in the second session.

At the end of this study period, participants' learning of the targets was assessed. To do so, we tested participants with studied views of the four targets shown one at a time in the middle of the computer monitor (the target images displayed during the study period were not present on the screen). Each target was shown three times, for a total of 12 trials $(3 \times 4$ targets) per block. Trial order was randomized, with the constraint that no target appeared more than twice in a row. Each trial began with a fixation cross at the center of the screen, which was accompanied by a short warning beep. A target replaced the fixation cross 2,300 ms later. The target remained on until the participant made a response. Four keys on the computer keyboard corresponding to $V, B, N$, and $M$ were designated as the response keys. The keys were labeled $1,2,3$, and 4 from left to right. Participants used the left middle finger, the left index finger, the right index finger, and the right middle finger to press the four keys, corresponding to Targets $1-4$, and were instructed to press the key that corresponded to the target presented. Accuracy as well as speed was emphasized in the instructions. An auditory beep was emitted for incorrect responses. An interval of $800 \mathrm{~ms}$ elapsed between the participant's response and the onset of the fixation cross for the next trial. The learning criterion was no more than one incorrect response per block in two consecutive blocks. The control participants met this criterion in the first two blocks of test trials. S.M. also met the criterion in the first two blocks of test trials in both of the sessions he completed; he was run in a third block of test trials to provide additional training.

\section{Test Phase}

The test phase consisted of two blocks. In the first test block, the test item was shown alone in the center of the computer screen and participants responded from memory by pressing the key corresponding to the learned target if it was a target or by pressing the spacebar if it was not a target. In the second block, icons of the studied items, arrayed in a row across the top of the monitor (as in the learning phase but without the response label), remained in view when the test item was shown in the center of the screen. The first and second test blocks are called no-icons and icons test blocks, respectively. The no-icons test block always preceded the icons block to serve as an undiluted test of memory at the start of the test phase. We included the icons test block to examine whether S.M.'s performance improved when reminders of the studied objects were present in the same display as the test item, making this more of a simultaneous match-tosample perceptual task.

\section{No-Icons Test Block}

On each trial, a target or a distractor was shown in the center of the screen. The icons depicting the targets were not present on the screen during these test trials (hence, the "no-icons" label). The four original targets were shown twice in each of six views: the studied view, the two picture-plane rotated views, and the three depth-rotated views (see Figure 3 ). Thus, there were 48 target trials ( 4 targets $\times 6$ views $\times 2$ ). The 16 distractors (8 PC distractors and $8 \mathrm{RC}$ distractors) were shown once in each of three picture-plane rotated views $\left(0^{\circ}, 90^{\circ}\right.$, and $\left.180^{\circ}\right)$, making a total of 48 distractor trials ( 16 distractors $\times 3$ rotations). Items were mixed and shown in random order. Before the test blocks began, participants were told that the targets might be shown from different viewpoints and that they might see objects other than the targets (distractors). Instructions stressed that targets should be identified by pressing the key corresponding to their number labels, as learned, regardless of the orientation in which they were shown. Participants were told to press the spacebar with their thumbs whenever a distractor appeared (four other fingers positioned over response keys, as above). In both test blocks, auditory feedback, a short beep, was given only when a mistake was made on a target presented in its studied view because such a mistake indicated forgetting of a learned target or not concentrating on the task.

\section{Icons Test Block}

The icons test block was identical to the no-icons test block except that the studied views of the four targets were always displayed along the top of the monitor but with no accompanying labels. The participants still had to match objects across different rotated views because the icons depicted each target only in its initially learned view. The icons test block allowed us to examine whether any impairments in distinguishing between the targets and the distractors could be overcome when the initial view of the target was in sight. Failure on this task would then indicate more of a perceptual than a memory problem.

\section{RESULTS}

\section{Study Phase}

\section{Control Participants}

In the initial assessment of learning at the end of the study phase (in which participants identified the four targets from the studied views only), control participants made no errors in the first block and $0.8 \%$ errors in the second block $(95 \%$ confidence interval $[\mathrm{CI}]$ $=0.0 \%-5.6 \%$ ). The mean RTs for each of the two blocks was 794 $\mathrm{ms}(95 \% \mathrm{CI}=556 \mathrm{~ms}-1,134 \mathrm{~ms})$ and $662 \mathrm{~ms}(95 \% \mathrm{CI}=494$ $\mathrm{ms}-889 \mathrm{~ms})$, respectively, reflecting faster classification in the second block.

\section{S.M.}

In the assessment of learning, S.M. made no errors in any of the three blocks. His mean RTs for the three blocks were 2,136 ms, $1,242 \mathrm{~ms}$, and $810 \mathrm{~ms}$ in Session 1 and $1,383 \mathrm{~ms}, 1,223 \mathrm{~ms}$, and $1,207 \mathrm{~ms}$ in Session 2. S.M. clearly identified the four targets slowly (he also needed more time in the initial study phase, see above), though his RT for the final block in Session 1 was within the $95 \%$ CI of the normal participants' Block 2 RTs. Of most relevance, however, is that he was ultimately able to learn to classify the objects from the studied view accurately (for another example of S.M.'s ability to acquire new visual representations, see Behrmann et al., 2005). Having demonstrated this acquisition, the question of interest now is whether the visual representations, derived during the learning phase, include a representation of the parts of the studied objects, the spatial relationships between them, or both. To address this question, we turn to the test blocks. We 
first compare S.M. and control participants on the different distractor types, assessing performance to part and relation changes between targets and distractors. We then examine performance on the targets, evaluating how S.M. and the control participants respond to changes in orientation from learned viewpoint.

\section{Test Phase \\ Distractors}

The measure of interest for the distractors is the falseidentification rate, indicating the acceptance of a distractor as a target. If false-identification rates are high, this will raise questions regarding the basis of highly accurate identification of targets (see the Targets section below). Moreover, observing whether PC or $\mathrm{RC}$ distractors are accepted as targets will indicate whether S.M. failed to represent the parts or the spatial relations in the learning phase. False-identification rates are shown in black in Figure 4.

\section{No-Icons Test Block}

Control participants. Control participants made relatively few false-identification errors to the PC and RC distractors, as shown in Table 2 and Figure 4, with marginally more false alarms to the latter than the former, $t(41)=1.978, p=.055$. The RT difference (1,073 ms vs. $1,105 \mathrm{~ms})$ for these two different distractor trials was not significant, however, $t(41)=1.287$, ns. Note that each distractor was presented in three picture-plane orientations. Because the $\mathrm{RC}$ distractors were all arbitrarily oriented with respect to the targets, examining the orientation effect would be meaningless. The nonrotated orientations of the PC distractors were aligned to those of the targets (see Figure 1), so it is possible to examine whether an orientation effect was found for the PC distractors with
Table 2

Responses to Distractors by Distractor Type and Orientation in the No-Icons Test Block

\begin{tabular}{lcc}
\hline & \multicolumn{3}{c}{ Distractor type } \\
\cline { 2 - 4 } \multicolumn{1}{c}{ Variable } & PC & RC \\
\hline Error mean (\%) & 1.6 & 3.2 \\
95\% CI (\%) & $0.0-8.5$ & $0.0-13.7$ \\
S.M. (\%) & 20.8 & 52.1 \\
RT mean (ms) & 1,073 & 1,105 \\
95\% CI (ms) & $636-1,809$ & $579-2,110$ \\
S.M. (ms) & 3,092 & 2,340 \\
\hline & \multicolumn{2}{c}{ PC distractor orientation } \\
\cline { 2 - 4 } & \multicolumn{3}{c}{$90^{\circ}$} \\
\hline RT mean (ms) & 1,060 & 1,076 \\
Error mean (\%) & 0.9 & 1.8 \\
\hline
\end{tabular}

Note. $\mathrm{PC}=$ part-changed distractor; $\mathrm{RC}=$ relation-changed distractor; $\mathrm{CI}=$ confidence interval; $\mathrm{RT}=$ response time.

respect to the studied orientations of the targets. There was no significant effect of orientation on error, $F(2,82)=0.415, n s$; or on RTs, $F(2,82)=1.310, n s$ (see Table 2).

S.M. S.M. falsely identified $21 \%(10 / 48)$ of the PC distractors and $52 \%(25 / 48)$ of the RC distractors (see Figure 4), both of which were beyond the upper $95 \%$ CI for the control participants, which were $8.5 \%$ and $13.7 \%$, respectively (see Table 2). Thus, S.M. was clearly impaired at rejecting both forms of distractors, relative to the controls, and was more impaired at rejecting the $\mathrm{RC}$ than PC distractors, $\chi^{2}(1, N=96)=10.1, p<.005$. For example,

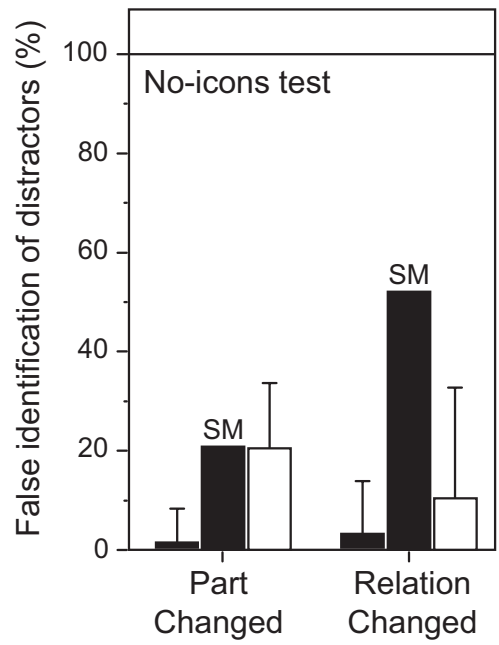

Distractor type

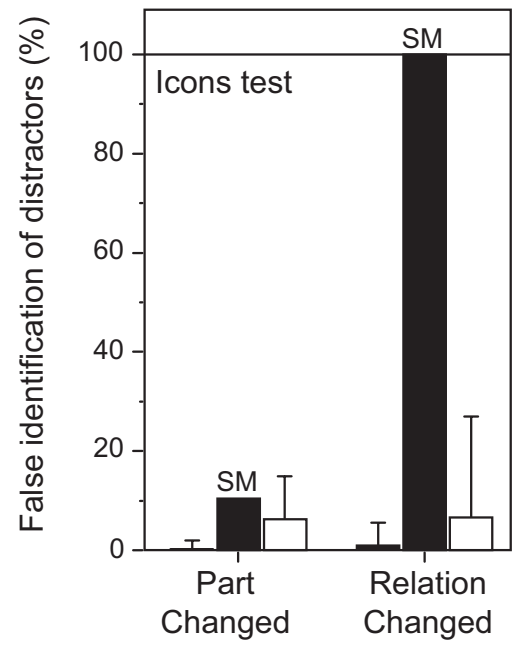

Distractor type

Figure 4. Percentage of false identifications of distractors for S.M. and normal participants. The falseidentification rates are plotted for the part-changed and the relation-changed distractors (no-icons block in the left panel and icons block in the right panel). The mean and 95\% confidence interval (CI) error bars of the data from the normal participants, collected under the same conditions as S.M., are solid. The mean and $95 \%$ CI error bars of the data from the normal participants, collected under brief exposure duration and masking, are open. 
although he did make errors rejecting a PC distractor such as D5 (see Figure 1B), in which the cube part of Target 1 (see Figure 1A) was changed to an ovoid part, he was more likely to consider an RC distractor such as D9 (see Figure 1C), in which the cube and cylinder parts of Target 1 were rearranged, to be equivalent to Target 1. S.M. also appears to have some difficulty representing the geon-like parts: In 5 of the 10 false identifications S.M. made to the PC distractors, the falsely identified targets did not share any part with the distractor. However, he has more trouble representing the spatial relationships between the geon-like parts: Of the 25 false identifications made to the RC distractors, all the falsely identified targets shared both parts with the distractors.

Although we have included S.M.'s RTs to PC and RC trials in Table 2, given the high error rate, these correct rejection RTs may be unstable and possibly meaningless, but the means are far slower than the $95 \%$ CI of the control participants.

\section{Icons Test Block}

Control participants. When the target icons were present (see Table 3), the control participants' false-identification rates were low for both types of distractors, although there were statistically more errors for RC $(1.0 \%)$ than for PC $(0.2 \%)$ trials, $t(41)=2.238$, $p<.05$. As above, there were no RT differences for the different distractor types, $t(41)=0.077, n s(582 \mathrm{~ms}$ vs. $583 \mathrm{~ms})$. Also, as above, the picture-plane rotation effect on the PC distractors was assessed with respect to the studied orientation of the targets. Although there was no significant effect of orientation on error, $F(2,82)=0.494, n s$, RTs were incrementally slower as degrees of orientation increased, $F(2,82)=4.072, p<.05$ (see Table 3 ).

S.M. Of interest, the greater false-identification rate for $\mathrm{RC}$ over PC was much exaggerated in the icons over the no-icons test block (see Figure 4). S.M. falsely identified very few of the PC distractors $(5 / 48$ or $10.4 \%)$ but all of the RC distractors $(48 / 48$ or $100 \%)$; this difference was clearly statistically significant, $\chi^{2}(1$, $N=96)=77.8, p<.0001$, and well beyond the CI of the control participants. In each of the five false identifications made to the PC

Table 3

Responses to Distractors by Distractor Type and Orientation in the Icons Test Block

\begin{tabular}{lcc}
\hline & \multicolumn{2}{c}{ Distractor type } \\
\cline { 2 - 3 } \multicolumn{1}{c}{ Variable } & PC & RC \\
\hline Error mean (\%) & 0.2 & 1.0 \\
95\% CI (\%) & $0.0-2.0$ & $0.0-5.6$ \\
S.M. (\%) & 10.4 & 100 \\
RT mean (ms) & 582 & 583 \\
95\% CI (ms) & $576-1,261$ & $522-1,394$ \\
S.M. (ms) & 4,297 & \\
\hline
\end{tabular}

\begin{tabular}{lccc}
\hline & \multicolumn{3}{c}{ PC distractor orientation } \\
\cline { 2 - 4 } & $0^{\circ}$ & $90^{\circ}$ & $180^{\circ}$ \\
\hline RT mean $(\mathrm{ms})$ & 829 & 856 & 873 \\
Error mean $(\%)$ & 0.3 & 0.0 & 0.3 \\
\hline
\end{tabular}

Note. $\quad \mathrm{PC}=$ part-changed distractor; $\mathrm{RC}=$ relation-changed distractor; $\mathrm{CI}=$ confidence interval; $\mathrm{RT}=$ response time. distractors, the falsely identified target always shared a part with the distractor. In each of the 48 false identifications made to the RC distractors, the falsely identified target always shared both parts with the misidentified distractor. That S.M. accepted the RC distractor in all cases but the PC in only a few instances is strongly suggestive of the claim that he has both geon parts available but that the binding of the parts is severely disordered.

\section{Can Task Difficulty Account for Distractor Error Pattern?}

On the basis of the findings with the distractors, we have argued that S.M. learned the parts of the two-geon displays, as reflected in the reduced error rate for PC distractors, but did not learn the relations between the parts, as evident from the high falseidentification rates for the RC distractors. It is interesting to note that this discrepancy between PC and RC false-alarm rates was greater in the icons than in the no-icons task, presumably because the icons served as lures for the parts of the two-geon displays. The poorer performance for the icons than the no-icons stands in contrast with the profile of the control participants, who were helped rather than hurt by the presence of the icons. Before examining S.M.'s performance on the targets, we need to consider an alternative interpretation of his performance with the distractors, in which the relative decrement for $\mathrm{RC}$ relative to $\mathrm{PC}$ is not attributable to an impairment in spatial relations per se but arises because the RC task is fundamentally more difficult than the PC task and that this difficulty is simply exaggerated in S.M. Unfortunately, we cannot evaluate this alternative with the current control data, as performance approaches ceiling on both PC and RC tasks.

To examine this alternative explanation on the basis of differences in task difficulty, we tested a second group of 11 control participants at Carnegie Mellon University, all of whom met the same inclusion criteria as the first set of participants. These participants completed the identical experiment, but now the exposure duration during the test phase was set to $100 \mathrm{~ms}$ and, immediately following the display, a pattern mask, composed of elements of a subset of the two-geon displays and covering the entire extent of the display on the screen, appeared and remained visible until response. These modifications of the paradigm were undertaken in an attempt to equate the false-identification rate of the normal controls to that of S.M. (21\%) on the PC distractors in the no-icons task, and the data from these participants are also shown in Figure 4. Three of the participants were excluded, 2 because their error rate was below 5\% even under these conditions and 1 whose error rate was $55 \%$. On the no-icons test, there was no significant difference between the mean error rate of the remaining control participants $(20.2 \%$; SD $=5.7 \%)$ and S.M. $(21 \%)$ on the PC distractors, $t(7)=1.613, p>.05 .{ }^{1}$ On the same test for the RC trials, the control participants made significantly fewer false identifications $(10.4 \% ; S D=9.5 \%)$ than S.M. $(52 \%), t(7)=4.364$, $p<.01$. Thus, S.M. was disproportionately impaired on the RC distractors in the no-icons task, even when the control participants' performance on the PC distractors was matched to that of

\footnotetext{
${ }^{1}$ Note that all comparisons of S.M. and these controls are done using the modified independent samples $t$-test method for comparing an individual's score with a normative sample (Crawford \& Howell, 1998).
} 
S.M. S.M.'s selective deficit on the RC distractors was even more apparent on the icons task: Whereas there was no statistical difference between the control participants $(M=6.2 \%, S D=3.7 \%)$ and S.M. $(10.4 \%)$ on PC trials, $t(7)=1.430, p>.05$, the controls made comparably fewer errors on RC trials $(M=6.6 \%, S D=$ $8.6 \%$ ) in contrast with S.M.'s $100 \%$ RC errors, $t(7)=10.3, p<$ .001 . The facts that (a) the control participants continue to perform relatively well on $\mathrm{RC}$ trials in the no-icons task even when performance is matched to S.M. on PC trials and (b) this pattern is even more exaggerated on the icons task suggest that S.M.'s profile cannot be accounted for simply by a difference in task difficulty for PC and RC trials. Instead, these data support the original claim that S.M. is able to learn to represent the parts of objects but not their relations.

\section{Does S.M.'s Pattern of Errors on Distractors Reflect a Limitation in Shape Perception?}

Our results suggest the possibility that S.M.'s brain damage impaired his ability to identify the spatial relationships between geon-like parts more than it impaired his ability to identify the geon-like parts themselves, even when their appearance was altered by changes in viewpoint. However, as discussed in the introduction, parts may be easier to identify than a whole either because of their simplicity or their numerosity. We therefore must consider the possibility that S.M.'s low rate of false identifications of the PC distractors and his high rate of false identifications of the RC distractors might have resulted because he initially encoded only one part from each object - the single-part-encoding hypothesis. When S.M. looked at Target 1 in Figure 1A, for example, did he encode both parts, "cube" and "cylinder," without encoding their spatial relationship, or did he encode only one part that appeared salient to him? If the latter were the case, then S.M.'s data would indicate a perceptual capacity limitation rather than an impaired ability to create or to access representations specifying the spatial relationships between the parts of the object.

The single-part encoding hypothesis predicts a lower percentage of errors with PC distractors than with RC distractors simply because only some of the former would contain one of the parts S.M. had encoded whereas all of the latter would. Accordingly, there would have been fewer chances for S.M. to identify the PC $(<48)$ than the RC (48) targets falsely, and error percentages would be underestimated when 48 was used as the denominator to calculate the percentage error in the PC conditions. There are 16 possible sets of single parts that S.M. might have encoded from the 4 two-part target objects $(2 \times 2 \times 2 \times 2=16)$. Depending on which of these sets he did encode, all 8 of the PC distractors contained at least one of the selected parts $(N=2$ sets of 4 parts; e.g., cube part from Target 1, pyramid part from Target 2 , brick part from Target 3, and ovoid part from Target 4), 6 of the PC distractors contained the selected parts $(N=12$ sets of 4 parts; e.g., cube part from Target 1, pyramid part from Target 2, U-shaped part from Target 3 , and cone part from Target 4$)$, or only 4 of the PC distractors contained the selected parts $(N=2$ sets of 4 parts; e.g., cube part from Target 1, flat part from Target 2, brick part from Target 3, and cone part from Target 4). If S.M. used the single-part-encoding strategy, then the appropriate denominators for calculating the error percentages for these different sets of single parts would have been 48 (if 8 PC distractors shared the selected target parts; i.e., $8 \times 3$ orientations $\times 2$ sessions), 36 (if 6 PC distractors shared the selected target parts), or 24 (if only 4 PC distractors shared the selected target parts), respectively.

We recomputed S.M.'s percentage of errors for the PC distractors using 24 as our denominator, the smallest denominator one might use to test the single-part encoding hypothesis. This constituted a liberal test of the single-part encoding hypothesis and a conservative test of the integration impairment hypothesis. If the single-part-encoding hypothesis were true, then the rate of partbased false identifications should become equivalent for the PC and RC distractors after implementing this liberal adjustment of the denominator for the PC distractors. On the contrary, the rate of part-based false identifications remained significantly higher for the RC distractors. In the no-icons test block, S.M.'s rate of part-based false identifications with the RC distractors $(52.1 \%$ or $25 / 48$ ) was still significantly higher than his adjusted rate of $20.8 \%$ $(5 / 24)$ with the PC distractors, $\chi^{2}(1, N=72)=6.4, p<.05$. This disparity was more pronounced in the icons test block; S.M. made $100 \%$ (48/48) part-based false identifications with the RC distractors as opposed to the adjusted rate of $20.8 \%(5 / 24)$ with the PC distractors, $\chi^{2}(1, N=72)=51.6, p<.0001$. Thus, despite the fact that the denominator for the PC distractors was chosen to optimize the single-part-encoding hypothesis, it fell significantly short of explaining the degree to which S.M. made disproportionately more false identifications of the RC distractors than of the PC distractors.

\section{Targets}

\section{No-Icons Test Block}

Control participants. As can be seen in Table 4 and Figure 5, for the picture-plane rotated views of the targets, control participants made equivalently few errors across the different rotations, $F(2,82)=1.086, n s$. As might be expected, the mean RT was

Table 4

Responses to Targets by Picture-Plane Rotation and Depth Rotation in the No-Icons Test Block

\begin{tabular}{|c|c|c|c|c|}
\hline \multirow[b]{2}{*}{ Variable } & \multicolumn{4}{|c|}{ Picture-plane rotation } \\
\hline & $0^{\circ}$ & & $90^{\circ}$ & $180^{\circ}$ \\
\hline Error mean $(\%)$ & 1.2 & & 2.4 & 1.5 \\
\hline $95 \%$ CI $(\%)$ & $0.0-8.7$ & & -13.9 & $0.0-11.5$ \\
\hline S.M. (\%) & 18.8 & & 18.8 & 25.0 \\
\hline RT mean (ms) & 968 & & ,129 & 1,178 \\
\hline $95 \%$ CI (ms) & $618-1,516$ & & $-1,823$ & $699-1,987$ \\
\hline \multirow[t]{3}{*}{ S.M. (ms) } & 1,636 & & 929 & 1,750 \\
\hline & \multicolumn{4}{|c|}{ Depth rotation } \\
\hline & $-30^{\circ}$ & $0^{\circ}$ & $30^{\circ}$ & $60^{\circ}$ \\
\hline Error mean $(\%)$ & 2.1 & 1.2 & 1.2 & 3.9 \\
\hline $95 \%$ CI $(\%)$ & $0.0-14.4$ & $0.0-8.7$ & $0.0-8.7$ & $0.0-18.1$ \\
\hline S.M. (\%) & 25.0 & 18.8 & 18.8 & 6.3 \\
\hline RT mean (ms) & 1,040 & 968 & 1,053 & 1,120 \\
\hline $95 \%$ CI (ms) & $670-1,616$ & $618-1,516$ & $673-1,649$ & $666-1,883$ \\
\hline S.M. (ms) & 2,042 & 1,636 & 1,726 & 2,200 \\
\hline
\end{tabular}

Note. $\quad \mathrm{CI}=$ confidence interval; $\mathrm{RT}=$ response time. 


\section{A. Picture-plane rotation}
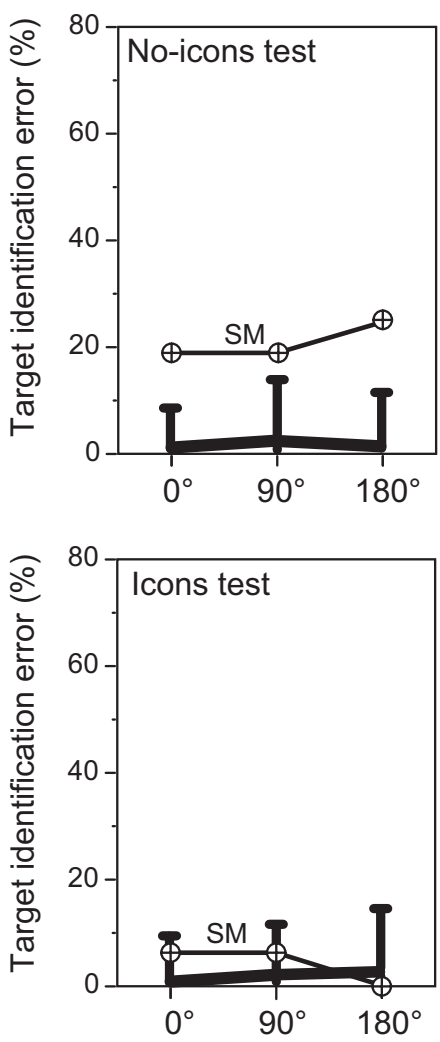

\section{B. Depth rotation}
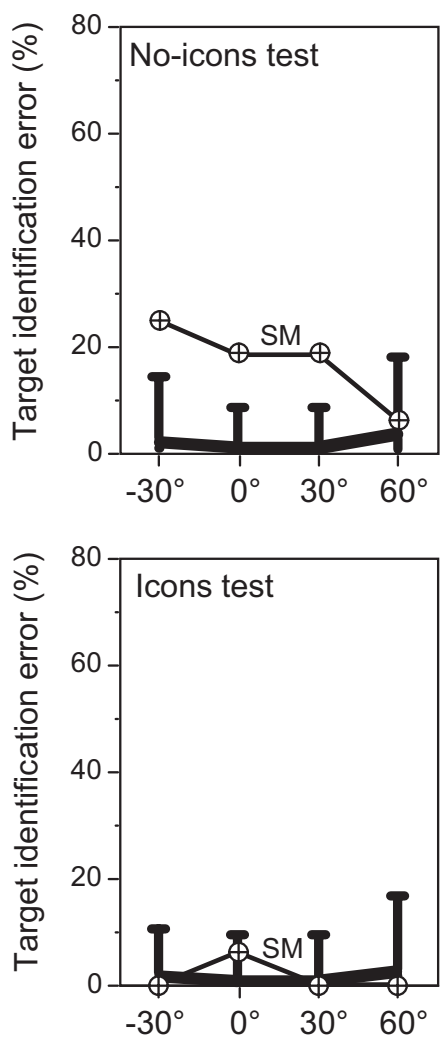

Figure 5. Percentage of target-identification errors for S.M. and normal participants for the picture-plane rotation (A) and the depth rotation (B). The top row is for the no-icons test block and the bottom row is for the icons test block. The mean of the normal control participants is shown by the thick black line. The error bars represent 95\% confidence intervals. S.M.'s error rates are shown in crossed circles.

slowed as picture-plane rotation increased, $F(2,82)=34.646, p<$ .0001 . For the depth-rotated views, the error differences were marginally significant, $F(3,123)=2.674, p=.0503$, with the largest number of errors for the largest $\left(60^{\circ}\right)$ rotation. Also, as expected, RT slowed incrementally as the degree of rotation in depth increased, $F(3,123)=13.331, p<.0001$, as shown in Table 4. Note that even though in all these trials control participants were identifying targets, the targets had not been seen in the non- $0^{\circ}$ orientations previously and so the participants were showing the predicted increment in identification as a function of degree of orientation change from the learned or familiar view (Hayward \& Tarr, 1997; Jolicoeur, 1985; Tarr \& Pinker, 1989).

S.M. S.M.'s error rates did not depend significantly on the picture-plane rotation, $\chi^{2}(1, N=48)=0.3, n s$, or on the depth rotation, $\chi^{2}(1, N=48)=2.1, n s$. Trial-based analyses show that S.M.'s mean RT also was not influenced significantly by pictureplane rotation, $F(2,35)=0.210, n s$, or by depth rotation, $F(3$, $49)=1.291, n s$. Compared with the control participants, S.M. was much less accurate in identifying the targets (see Figure 5). In the context of the distractors, he identified the studied view $81.2 \%$ of the time. Note that, although this is less accurate than the controls in the no-icons test block, he correctly identified targets significantly more often than he falsely identified RC distractors, $\chi^{2}(1$,
$N=48)=4.2, p<.05$. These data suggest that S.M.'s memory representation of the spatial relationships between the geon-like parts was impaired but not entirely absent.

\section{Icons Test Block}

Control participants. The control individuals identified targets extremely well both in the picture-plane and depth rotations, with fewer than $3 \%$ error rates in even the least accurate condition (see Table 5 and Figure 5). The extent of the rotation did not affect accuracy for either picture-plane or depth rotation: picture plane, $F(2,82)=1.414, n s ;$ depth, $F(3,123)=1.229, n s$. However, in both cases, RT scaled with the degree of rotation: picture plane, $F(2,82)=39.978, p<.0001 ;$ depth rotation, $F(3,123)=13.331$, $p<.0001$.

S.M. S.M.'s performance was slow but accurate. His RT was slower than that of the control participants and fell outside the $95 \%$ CI for every degree of orientation for both types of rotations. S.M.'s RTs depended marginally on the picture-plane orientation, $F(2,43)=3.071, p<.057$, but did not depend on the depth orientation, $F(3,59)=0.571, n s$. His error rates were very low and showed no significant orientation dependency: $\chi^{2}(1, N=48)=$ 
Table 5

Responses to Targets by Picture-Plane Rotation and Depth Rotation in the Icons Test Block

\begin{tabular}{|c|c|c|c|c|}
\hline \multirow[b]{2}{*}{ Variable } & \multicolumn{4}{|c|}{ Picture-plane rotation } \\
\hline & \multicolumn{2}{|l|}{$0^{\circ}$} & $90^{\circ}$ & $180^{\circ}$ \\
\hline Error mean $(\%)$ & \multicolumn{2}{|l|}{0.9} & 2.1 & 2.7 \\
\hline $95 \%$ CI $(\%)$ & $0.0-9.5$ & \multicolumn{2}{|c|}{$0.0-11.6$} & $0.0-14.6$ \\
\hline S.M. (\%) & \multicolumn{2}{|l|}{6.3} & 6.3 & 0.0 \\
\hline RT mean (ms) & 812 & \multicolumn{2}{|c|}{904} & 964 \\
\hline $95 \%$ CI (ms) & \multicolumn{3}{|c|}{$581-1,405$} & $584-1,594$ \\
\hline \multirow[t]{3}{*}{ S.M. (ms) } & 2,145 & \multicolumn{2}{|c|}{2,434} & 1,717 \\
\hline & \multicolumn{4}{|c|}{ Depth rotation } \\
\hline & $-30^{\circ}$ & $0^{\circ}$ & $30^{\circ}$ & $60^{\circ}$ \\
\hline Error mean $(\%)$ & 1.8 & 0.9 & 0.9 & 2.7 \\
\hline $95 \%$ CI $(\%)$ & $0.0-10.7$ & $0.0-9.5$ & $0.0-9.5$ & $0.0-16.9$ \\
\hline S.M. (\%) & 0.0 & 6.3 & 0.0 & 0.0 \\
\hline RT mean (ms) & 845 & 812 & 870 & 930 \\
\hline $95 \%$ CI (ms) & $567-1,260$ & $543-1,216$ & $572-1,326$ & $555-1,559$ \\
\hline S.M. (ms) & 1,896 & 2,145 & 2,133 & 2,248 \\
\hline
\end{tabular}

Note. $\quad \mathrm{CI}=$ confidence interval; $\mathrm{RT}=$ response time

1.0, $n s$, for the picture-plane rotation, and $\chi^{2}(1, N=48)=3.0, n s$, for the depth rotation.

There is reason to believe that S.M.'s relatively high accuracy with targets may have been accomplished by his simply matching the parts against the icons; that is, by using the same strategy that led him to falsely identify all of the RC distractors as targets. Of interest, when we count as correct any response in which S.M. identified a target or an RC distractor solely on the basis of matching parts, we find that he was extremely accurate: $142 / 144$ or $98.6 \%$ correct identification (where chance is $20 \%$-five possible responses, i.e., any of the four targets or a distractor). Thus, it seems that S.M. could match pairs of geon parts in the targets to pairs of geon parts in the icons across rotations in the picture plane and in depth. Although his RT was slower than that of the controls and fell outside the $95 \%$ CI for every degree of orientation for both rotations, he was able to perform identification with good accuracy. S.M.'s relatively good performance on this task is consistent with the finding that he can match objects with foreshortened axes. That he can represent geon parts flexibly enough to generalize over viewpoint, rather than depend only on a representation of the geon part from the studied vantage point, is interesting and suggests that, unlike some other individuals with agnosia (Warrington, 1982), his object perception deficit is specific to a problem in representing spatial relations between components rather than to a more general visual impairment.

One final analysis was conducted to confirm that S.M.'s selective deficit on the RC distractors could not be attributable to differences in part orientations between the PC and RC distractors. For example, one might argue that the RC distractors D9-D12 could have been particularly difficult to reject because when these distractors were shown in unrotated orientations (as shown in Figure 1), all of their parts were in the same orientation as the corresponding parts of the learned targets. This possibility was tested by comparing S.M.'s performance on D9-D12 with his performance on the remaining RC distractors (D13-D16) because no parts of the unrotated versions of these latter RC distractors had the same orientation as any parts of the learned targets. Thus, if S.M.'s overall elevated error on the RC distractors was due to alignment of part orientation with the learned targets, S.M. would have made substantially more errors on the unrotated D9-D12 than on the unrotated D13-D16. Contrary to this prediction, S.M. made an identical number of errors on both types of distractors (4 out of 8 a piece on the no-icons task and 8 out of 8 a piece on the icons task). Differences in part orientation thus cannot explain S.M.'s elevated errors on the RC distractors. This demonstration of S.M.'s indifference to part orientation in rejecting the RC distractors combined with his overall $98.6 \%$ "correct" performance across orientation changes when all successful part-based matches are counted as correct further endorse our claim that S.M. was well capable of view-invariant processing of volumetric parts within two-part objects.

\section{DISCUSSION}

The goal of this article was to examine the processes involved in the internal representation of object structure. More specifically, this study was designed to explore the claim that objects are represented as a collection of constituent parts and that the spatial relations by which these parts are integrated is coded independently of the parts themselves. The approach adopted was to assess whether an individual who has been shown previously to be impaired at deriving a global whole has available the local components of objects but not the relations between them. Patient S.M., previously diagnosed as having IA, and a large group of control participants learned to identify four novel objects, each made of two unique local parts. Previous results using common objects and novel multipart objects suggested that S.M. could only encode a small number of parts and/or had trouble encoding spatial arrangements of parts. To contrast these possibilities, we evaluated his ability to identify individual geons within two-part objects and his ability to identify the spatial arrangements of pairs of geon parts as follows: Once the targets were learned, they were presented either from the learned viewpoint or from a different viewpoint where the rotation could be in the picture plane or in the depth plane. In addition, two types of distractors were presented: those that shared parts with the target but not the manner of attachment of those parts (RC distractors) and those that did not share the parts (PC distractors). These stimuli were presented either with the targets present at the top of the screen as an iconic reference or in the absence of any prompt, thereby requiring reliance on stored internal representation.

The findings revealed that all control participants learned the four target items well. Moreover, these participants made few errors when the targets appeared in new orientations, in the frontal plane or in depth, and equally so for memory (no icons) and perceptual (icons) matching. As expected from many existing studies, rotations in the picture plane as well as relatively small rotations in depth around the vertical axis slowed identification of the targets for the normal participants (Johnston \& Hayes, 2000; Tarr, Bülthoff, Zabinski, \& Blanz, 1997), with systematic decrement in performance as the view of the object to be recognized deviates from its familiar view. This was true as targets were rotated further from the studied view both in the picture plane or 
in depth about the vertical axis, consistent with the body of work demonstrating costs to recognition performance associated with changes in viewpoint (Hayward, Wong, \& Spehar, 2005; Humphrey \& Khan, 1992; Tarr et al., 1997; Tarr \& Pinker, 1989). The target-distractor discrimination posed minimal difficulty for the normal participants, regardless of whether the distractors had to be discriminated on the basis of single mismatching parts or on the basis of differences in spatial arrangements of the two parts, and this was true in the icons and no-icons task. It was also the case that performance was relatively good even under brief masked presentation, especially on the icons trials.

S.M. was also able to learn the targets, albeit with more extended practice than the control participants. At test, his identification of targets in new orientations was somewhat error prone for no-icons matching, when the learned stimuli were not visible, but his performance was within the normal range for icons matching, when the learned stimuli were present on the screen. For S.M., when it was possible to perform the task by comparing individual geon parts (target identification and rejection of PC distractors), he performed well regardless of the picture-plane and depth rotations used, especially in the presence of the icons. This contrasts with the findings from patients with agnosia who either show excessive reliance on viewpoint dependence (Farah \& Hammond, 1988; Warrington \& James, 1988) or from those who have orientation agnosia, with preserved recognition of stimuli rotated in the picture plane but impaired recognition of a picture's orientation (e.g., naming a picture of a bus correctly but copying the bus in an upside-down orientation; Turnbull, 1997; Turnbull, Laws, \& McCarthy, 1995).

In contrast with this relative preservation of viewpoint independence for geon parts, when discrimination of spatial arrangements was required (rejection of RC distractors), S.M. performed partbased matching correctly while being severely impaired at discriminating salient differences in the spatial arrangements of two parts, identifying the majority of the RC distractors as the targets that have the same pair of parts. Our data allowed us to reject the possibility that S.M. had encoded only one part from each two-part object while ignoring the other part. S.M.'s performance could also not be explained merely by the possibility that the spatial relations distractors were more difficult than the part relations distractors. Taken together, the results suggest that S.M. was indeed able to encode both parts in the two-part objects but that he was severely impaired at encoding the spatial arrangement of the two parts. In short, he represented objects as a collection of parts, which he could recognize from various views but without regard to the spatial relations between the parts.

It is interesting to note that the individual parts (or geons) used here were also specific spatial arrangements of simple image features, such as straight and curved edges and their intersections. It thus appears that S.M. was able to discriminate spatial arrangements of image features forming different geons but that his ability to process spatial relationships broke down dramatically at the level of discriminating spatial arrangements of a pair of geons.

The finding that S.M. retained some ability to discriminate the $\mathrm{RC}$ distractors from the targets when no icons were present but was completely unable to reject the RC distractors when the icons were present was also of interest. In the no-icons block, an identification response required a match between the test item and a memory representation of the learned item. Perhaps the details of the learned items, such as their part decomposition and individual contours, were obscured in S.M.'s memory representation. S.M. then might have relied more on global aspects of the targets, such as overall outlines, enabling him to reject some of the RC distractors. In contrast, when S.M. compared test objects and targets side by side in the icons block, his unusual partwise perceptual strategy might have been engaged obligatorily. This possibility is consistent with the fact that S.M.'s ability to reject the RC distractors dramatically worsened in the icons block but his ability to reject the PC distractors on the basis of mismatching parts, reflecting his ability to compare individual parts, improved in the icons block. (His correct identifications of the targets also increased in the icons block, as did his false identifications of the RC distractors. Both of these behaviors might reflect a part-matching approach.)

Another related possibility is that memory for parts is worse when they cannot be integrated into a whole. In the no-icon (memory) condition, S.M. is forced to retain individual parts without their being integrated, making his memory load effectively larger than that of controls, much as memory for a group of unrelated numbers is worse than when the words are chunked into a known sequence. Consequently, he forgets exactly what the target is, leading to relatively increased errors on the PC condition and relatively decreased false alarms on the $\mathrm{RC}$ condition because he simply does not remember the target well enough to appreciate that the distractor is a rotated version of it. When the target is present in the icons condition, his performance on the PC distractors improves but performance on the RC distractors worsens because all the parts that make up the target are apparent but he cannot appreciate the differences in their spatial arrangement, forcing him to rely on a part-by-part comparison to determine a match.

Obligatory engagement of a defective perceptual strategy has previously been demonstrated in an individual with prosopagnosia, L.H., who is selectively impaired at face recognition. L.H. matched upside-down faces more accurately than upright faces (this is true for H.J.A. as well and, to some extent, for S.M.), suggesting that a defective face-processing mechanism was engaged in an obligatory manner when upright faces were presented, whereas when upside-down faces were presented, other image comparison strategies were no longer preempted by the defective mechanism because upside-down faces do not strongly engage face-specific mechanisms (de Gelder, Bachoud-Levi, \& Degos, 1998; Farah, Wilson, Drain, \& Tanaka, 1995).

Taken together, these results support the hypothesis that S.M.'s brain damage did not impair his ability to identify parts of the test objects but impaired his ability to identify the spatial relationships between them. It is important to note that we were able to reject the possibility that a general shape recognition deficit could account for our results. We were also able to show that S.M. was less impaired in integrating the features of the individual parts than he was in representing the spatial relationships among the parts. Thus, these findings are compatible with a view in which independent, and hence dissociable, processes mediate the perception of parts and of the relations between the parts. These data are also consistent with the findings from a previous report documenting the decrement in performance of Patient H.J.A. on tasks requiring judgments based on the spatial relations between the local parts of objects with relative preservation of the object parts themselves (Riddoch, Humphreys, Blott, Hardy, \& Smith, 2003). H.J.A.'s 
deficit was also more apparent when the global outline of the final pattern was complex and when the time to encode the elements was reduced. Our results are also compatible with the data from Patient R.K., who was impaired at object recognition, especially when objects were shown from unconventional views (Davidoff \& Warrington, 1999). Although R.K. was impaired both at recognizing objects and their spatial relations, he did show some ability to recognize object parts from canonical views and this finding provides some additional support for the separation between parts and their spatial framework.

\section{RELATIONSHIP TO OTHER RESEARCH}

We have suggested that both part and spatial relation processing are critical for object recognition, and the data reviewed in the introduction argued for a developmental time course during which whole-based processing is only evident in older children whereas part-based processing predominates in younger children. In some adult studies, however, the ability to identify spatial relationships has been shown to play an early and perhaps greater role in object perception than the discrimination of the individual features of objects: For example, Johnston and Hayes (2000) had participants perform both a sequential matching task and a learning task with objects composed of three geons. The objects could all be made of different local parts but share the spatial arrangements or could be composed of the same three parts but in different spatial configurations. Recognition of the former was considerably more difficult than that of the latter-when different spatial relationships are used, the outline shape of each object becomes completely distinct from all others and relatively early visual processes may be able to assist in recognition judgments. Although these findings support the idea that spatial relations of parts are perhaps more readily identified than the properties of parts themselves, because the experimental design does not orthogonally manipulate local parts and spatial relations, their results cannot definitively ascertain the relative contribution of parts and relations in object recognition or their potential independence.

Related work conducted by Arguin and Saumier (2004; Saumier, Arguin, Lefebvre, \& Lassonde, 2002) examined these issues further. In this work, the authors independently manipulated the parts and their spatial relations within each object and examined how these manipulations influenced visual search performance. Their observers searched for a three-part target consisting of three volumetric geons arranged in a particular spatial relationship embedded among distractors that were composed of either the same or different parts and had either the same or a different spatial organization of the parts as the target. Arguin and Saumier (2004) observed a performance cost both when the target and distractors shared parts and when they shared spatial organization, but they did not obtain a statistically significant interaction between part sharing and organization sharing. Using additive factors logic, they took these data to indicate that parts and their spatial organization made independent contributions to visual search performance. The absence of an interaction is a null effect, however, and caution must be applied when arguing from null effects. In Arguin and Saumier's (2004) case, the interaction term was marginally significant $(p=.08)$ and the number of participants was small $(N=12)$. Moreover, when Saumier et al. (2002) used this same procedure with 10 other individuals as well as with a patient with agnosia, the data again suggested an interaction between parts and spatial organization for both the patient and the control participants (although the statistical significance could not be assessed because these conditions were not entered into the analyses of variance as separate factors). These potential interaction effects might also have occurred because the global structure of the distractors and targets might have been especially similar when they shared both parts and part relations in the same organization condition. Thus, because the studies by Arguin and Saumier (2004) and Saumier et al. (2002) are suggestive of weakly interacting contributions from processing of parts and spatial relations in visual search, they do not definitively resolve the question of whether parts and spatial relations can be encoded independently by neuropsychologically separable neural substrates.

Evidence suggestive of separate part and relations coding is also obtained from other neuropsychological data. For example, Patient G.K., whose visual errors often corresponded to identifying a part as an object (e.g., antler $\rightarrow$ "deer;" ladder $\rightarrow$ "fire engine") was impaired at discriminating global shape, especially when the local components were closed, that is, suggestive of objects unto themselves (Humphreys et al., 1994). Undue reliance on the parts is also revealed in the errors of individuals with IA: Patient H.J.A., for example, misidentified the snout of a seahorse as a "bird's beak," and his error was not remedied by his perception of the rest of the seahorse's image (Ballaz, Boutsen, Peyrin, Humphreys, \& Marendaz, 2005). Patient C.K. also reveals errors based on local components, calling a dart a "feather duster" and a tennis racquet a "fencer's mask" (Behrmann et al., 1994; Behrmann, Winocur, \& Moscovitch, 1992). Because of the design of our experiment, S.M.'s complete failure at discriminating spatial relations, and the method we used to rule out a single-part-encoding explanation for S.M.'s performance, our results go beyond these other findings to show more clearly the separability of part and relations coding.

A similar argument in which object processing requires not only the local features but also their spatial relations is made in the context of face processing, with the reliance on the spatial relations perhaps even more critical (Leder \& Bruce, 2000; Maurer, Le Grand, \& Mondloch, 2002; Tanaka \& Farah, 1993). As with the object processing, a similar developmental trend has been suggested for face processing, with an earlier reliance on the processing of parts and the representation of the spatial relations into a configuration with increasing age (Carey \& Diamond, 1994; Chung \& Thomson, 1995). Of interest is that S.M. is also impaired at face processing, a finding that is consistent with this failure to extract the spatial relations between local components and derive the configuration (Behrmann \& Kimchi, 2003; Behrmann et al., 2005). The findings that object representations rely not only on parts but also on their relations and that this extends to faces, with perhaps even greater reliance on the configuration for faces, suggest that the parallel processing of these two sources of visual information is a general principle subserving multiple domains of object recognition. Note that this issue has also been considered in the context of word reading, and letter-by-letter reading is thought to be a paradigmatic case of reliance on part-based processing (Behrmann, Shomstein, Barton, \& Black, 2001; Farah, 1992).

Taken together, our data and the neuropsychological data discussed above suggest that the internal representation of objects consists of separate codes for the parts and for the relations between these parts and that these two codes can be selectively 
disrupted. Although we have argued in favor of independent processing of parts and their relations, this does not mean that these two sources of information cannot interact. Bottom-up representation of the parts can certainly be aided in a top-down interactive fashion from preexisting representation of objects, and this feedback can facilitate processing of local elements or parts and assist in the recovery of the local components under degraded conditions (Moore \& Cavanagh, 1998; Weisstein \& Harris, 1974; Wheeler, 1970). In addition, object processing can be affected by structural properties that go beyond the combination of local parts; for instance, emergent features such as "goodness" of parts can be determined reliably on the basis of interrelationships of parts of the object (Palmer, 1977, 1978). Finally, single unit recordings with awake behaving monkeys have demonstrated that although some neurons have selective neural activity for specific parts of an object, other neurons respond nonlinearly to specific combination of parts, further attesting to the interactivity of the component parts in reflecting object structure (Baker, Behrmann, \& Olson, 2002).

\section{CONCLUSION}

In conclusion, a patient with visual object agnosia, S.M., was able to learn to identify simple volumetric objects made of two discrete geons. At test, identification difficulty was manipulated by presenting the targets in various rotated views and by intermixing distractors. The most dramatic finding was that S.M. was severely impaired at discriminating distractors on the basis of mismatching spatial arrangements. This cannot be attributed to his failure to learn both parts initially, as his results cannot be explained by a single-part-encoding hypothesis and, moreover, he made few errors when a single part of the distractor was switched with the target. This disproportionate difficulty is also not attributable to increased task difficulty because the objects consisted of only two parts and the differences in spatial arrangements were salient (the normal participants rarely made false identifications) and because normal participants performed well on spatial relations trials when performance on part relations trials was equated to that of S.M. Also, these relation-based confusions occurred more often when the targets were present on the screen and acted as perceptual references and no long-term representation was needed. These findings thus suggest that discriminating spatial relationships between simple volumetric parts requires a mechanism that is separate from that required for identifying specific arrangements of image features (e.g., edges, edge junctions, and their spatial relations) that define simple volumes.

\section{References}

Arguin, M., \& Saumier, D. (2004). Independent processing of parts and their spatial organization in complex objects. Psychological Science, 15, 629-633.

Baker, C., Behrmann, M., \& Olson, C. (2002). Impact of visual discrimination training on the representation of parts and wholes in monkey inferotemporal cortex. Nature Neuroscience, 5, 1210-1216.

Ballaz, C., Boutsen, L., Peyrin, C., Humphreys, G. W., \& Marendaz, C. (2005). Visual search for object orientation can be modulated by canonical orientation. Journal of Experimental Psychology: Human Perception and Performance, 31, 20-39.

Behrmann, M., \& Kimchi, R. (2003). What does visual agnosia tell us about perceptual organization and its relationship to object perception?
Journal of Experimental Psychology: Human Perception and Performance, 29, 19-42.

Behrmann, M., Marotta, J., Gauthier, I., Tarr, M. J., \& McKeeff, T. J. (2005). Behavioral change and its neural correlates in visual agnosia after expertise training. Journal of Cognitive Neuroscience, 17, 554 568.

Behrmann, M., Moscovitch, M., \& Winocur, G. (1994). Intact visual imagery and impaired visual perception in a patient with visual agnosia. Journal of Experimental Psychology: Human Perception and Performance, 20, 1068-1087.

Behrmann, M., Shomstein, S., Barton, J. J., \& Black, S. E. (2001). Eye movements reveal the sequential processing in letter-by-letter reading. Neuropsychologia, 39, 983-1002.

Behrmann, M., Winocur, G., \& Moscovitch, M. (1992, October 15). Dissociations between mental imagery and object recognition in a braindamaged patient. Nature, 359, 636-637.

Benton, A. L., Hamsher, K., Varney, N. R., \& Spreen, O. (1978). Facial recognition: Stimulus and multiple choice pictures. New York: Oxford University Press.

Benton, A. L., Sivan, A. B., Hamsher, K., Varney, N. R., \& Spreen, O. (1983). Contributions to neuropsychological assessment (2nd ed.). Oxford, England: Oxford University Press.

Biederman, I. (1987). Recognition-by-components: A theory of human image understanding. Psychological Review, 94, 115-147.

Biederman, I., Subramaniam, S., Bar, M., Kalocsai, P., \& Fiser, J. (1999). Subordinate-level object classification reexamined. Psychological Research, 62, 131-153.

Binford, T. O. (1971, December). Visual perception by computer. Paper presented at the IEEE Systems Science and Cybernetics Conference. Miami, FL.

Black, S. E., Vu, B., Martin, D., \& Szalai, J. P. (1990). Evaluation of a bedside battery for hemispatial neglect in acute stroke [Abstract]. Journal of Clinical and Experimental Neuropsychology, 12, 102.

Butter, C. M., \& Trobe, J. D. (1994). Integrative agnosia following progressive multifocal leukoencephalopathy. Cortex, 30, 145-158.

Carey, S., \& Diamond, R. (1994). Are faces perceived as configurations more by adults than by children? Visual Cognition, 1, 253-274.

Chainay, H., \& Humphreys, G. W. (2001). The real object advantage in agnosia: Evidence for a role of shading and depth in object recognition. Cognitive Neuropsychology, 12, 175-191.

Chung, M. S., \& Thomson, D. M. (1995). Development of face recognition. British Journal of Psychology, 86, 55-87.

Coslett, H. B., Stark, M., Rajaram, S., \& Saffran, E. M. (1996). Narrowing the spotlight: A visual attentional disorder in Alzheimer's disease. $\mathrm{Neu}$ rocase, $1,305-318$.

Crawford, J. R., \& Howell, D. C. (1998). Comparing an individual's test scores against norms derived from small samples. The Clinical Neuropsychologist, 12, 482-486.

Davidoff, J., \& Roberson, D. (2002). Development of animal recognition: A difference between parts and wholes. Journal of Experimental Child Psychology, 81, 217-234.

Davidoff, J., \& Warrington, E. K. (1999). The bare bones of object recognition: Implications from a case of object recognition impairment. Neuropsychologia, 37, 279-292.

de Gelder, B., Bachoud-Levi, A. C., \& Degos, J. D. (1998). Inversion superiority in visual agnosia may be common to a variety of orientationpolarised objects besides faces. Vision Research, 38, 2855-2861.

Edelman, S. (1997). Computational theories of object recognition. Trends in Cognitive Science, 1, 296-304.

Efron, R. (1968). What is perception? Boston Studies in Philosophy of Science, 4, 137-173.

Farah, M. J. (1990). Visual agnosia: Disorders of object recognition and what they tell us about normal vision. Cambridge, MA: MIT Press.

Farah, M. J. (1992). Is an object an object an object? Cognitive and 
neuropsychological investigations of domain specificity in visual object recognition. Current Directions in Psychological Science, 1, 164-169.

Farah, M. J. (2004). Visual agnosia (2nd ed.). Cambridge, MA: MIT Press. Farah, M. J., \& Hammond, K. M. (1988). Mental rotation and orientationinvariant object recognition: Dissociable processes. Cognition, 29, 2946.

Farah, M. J., Wilson, K. D., Drain, H. M., \& Tanaka, J. R. (1995). The inverted face inversion effect in prosopagnosia: Evidence for mandatory, face-specific perceptual mechanisms. Vision Research, 35, 2089-2093.

Foster, D. H., \& Gilson, S. J. (2002). Recognizing novel three-dimensional objects by summing signals from parts and views. Proceedings in Biological Science, 269, 1939-1947.

Gauthier, I., Behrmann, M., \& Tarr, M. J. (1999). Can face recognition really be dissociated from object recognition? Journal of Cognitive Neuroscience, 11, 349-370.

Gauthier, I., Behrmann, M., \& Tarr, M. J. (2004). Are greebles like faces? Using the neuropsychological exception to test the rule. Neuropsychologia, 42, 1961-1970.

Giersch, A., Humphreys, G., Boucart, M., \& Kovács, I. (2000). The computation of occluded contours in visual agnosia: Evidence for early computation prior to shape binding and figure-ground coding. Cognitive Neuropsychology, 17, 731-759.

Goodglass, H., Kaplan, E., \& Weintraub, S. (1983). Boston Naming Test. New York: Lea \& Febiger.

Grailet, J. M., Seron, X., Bruyer, R., Coyette, F., \& Frederix, M. (1990). Case report of a visual integrative agnosia. Cognitive Neuropsychology, 7, 275-309.

Hayward, W. G., \& Tarr, M. J. (1997). Testing conditions for viewpoint invariance in object recognition. Journal of Experimental Psychology: Human Perception and Performance, 23, 1511-1521.

Hayward, W. G., Wong, A. C. N., \& Spehar, B. (2005). When are viewpoint costs greater for silhouettes than for shaded images? Psychonomic Bulletin \& Review, 12, 321-327.

Hoffman, D. D., \& Richards, W. A. (1984). Parts of recognition. Cognition, 18, 65-96.

Hummel, J. (1994). Reference frames and relations in computational models of object recognition. Current Directions in Psychological Science, 3, 111-116.

Hummel, J. E. (2001). Complementary solutions to the binding problem in vision: Implications for shape perception and object recognition. Visual Cognition, 8, 489-517.

Hummel, J. E., \& Biederman, I. (1992). Dynamic binding in a neural network for shape recognition. Psychological Review, 99, 480-517.

Hummel, J. E., \& Stankiewicz, B. J. (1996). An architecture for rapid, hierarchical and structural description. In T. Inui \& J. L. McClelland (Eds.), Attention and performance XVI: Information integration in perception and communication (pp. 93-121). Cambridge, MA: MIT Press.

Hummel, J. E., \& Stankiewicz, B. J. (1998). Two roles for attention in shape perception and structural-description model of visual scrutiny. Visual Cognition, 5, 49-79.

Humphrey, G. K., Goodale, M. A., Jakobson, L. S., \& Servos, P. (1994). The role of surface information in object recognition: Studies of a visual form agnosic and normal subjects. Perception, 23, 1457-1481.

Humphrey, G. K., \& Khan, S. C. (1992). Recognizing novel views of three-dimensional objects. Canadian Journal of Experimental Psychology, 46, 170-191.

Humphreys, G. W. (1999). Integrative agnosia. In G. W. Humphreys (Ed.), Case studies in vision (pp. 41-58). London: Psychology Press.

Humphreys, G. W., \& Riddoch, M. J. (1987). To see but not to see: A case study of visual agnosia. Hillsdale, NJ: Erlbaum.

Humphreys, G. W., \& Riddoch, M. J. (2001). Neuropsychological disorders of visual object recognition and naming. In F. Boller \& J. Grafman (Eds.), Handbook of neuropsychology (Vol. 4, pp. 159-180). Amsterdam: Elsevier Science.
Humphreys, G. W., \& Riddoch, M. J. (2006). Features, objects, action: The cognitive neuropsychology of visual object processing, 1984-2004. Cognitive Neuropsychology, 23, 1-28.

Humphreys, G. W., Riddoch, M. J., Donnelly, N., Freeman, T., Boucart, M., \& Muller, H. M. (1994). Intermediate visual processing and visual agnosia. In M. J. Farah \& G. Ratcliff (Eds.), The neuropsychology of high-level vision (pp. 63-101). Hillsdale, NJ: Erlbaum.

Humphreys, G. W., Riddoch, M. J., \& Quinlan, P. T. (1985). Interactive processes in perceptual organization: Evidence from visual agnosia. In M. I. Posner \& O. S. Marin (Eds.), Attention and performance XI (pp. 301-318). Hillsdale, NJ: Erlbaum.

Humphreys, G. W., Riddoch, M. J., Quinlan, P. T., Price, C. J., \& Donnelly, N. (1992). Parallel pattern processing and visual agnosia. Canadian Journal of Psychology, 46, 377-416.

Humphreys, K., Avidan, G., \& Behrmann, M. (in press). A detailed investigation of facial expression processing in congenital prosopagnosia as compared to acquired prosopagnosia. Experimental Brain Research.

Jankowiak, J., Kinsbourne, M., Shalev, R. S., \& Bachman, D. L. (1992). Preserved visual imagery and categorization in a case of associative visual agnosia. Journal of Cognitive Neuroscience, 4, 119-131.

Johnston, M. B., \& Hayes, A. (2000). An experimental comparison of viewpoint-specific and viewpoint-independent models of object representation. Quarterly Journal of Experimental Psychology: Human Experimental Psychology, 53(A), 792-824.

Jolicoeur, P. (1985). The time to name disoriented natural objects. Memory \& Cognition, 13, 289-303.

Kaplan, E., Goodglass, H., \& Weintraub, S. (1976). Boston Naming Test. Boston: Veterans Administration Hospital.

Kartsounis, L., \& Warrington, E. K. (1991). Failure of object recognition due to a breakdown in figure-ground discrimination in a patient with normal acuity. Neuropsychologia, 29, 969-980.

Leder, H., \& Bruce, V. (2000). When inverted faces are recognized: The role of configural information in face recognition. Quarterly Journal of Experimental Psychology: Human Experimental Psychology, 53(A), 513-536.

Marr, D., \& Nishihara, H. K. (1978). Representation and recognition of the spatial organization of three-dimensional shapes. Proceedings of the Royal Society of London, Series B, 200, 269-294.

Maurer, D., Le Grand, R., \& Mondloch, C. J. (2002). The many faces of configural processing. Trends in Cognitive Sciences, 6, 255-260.

Moore, C., \& Cavanagh, P. (1998). Recovery of 3D volume from 2-tone images of novel objects. Cognition, 67, 45-71.

Palmer, S. E. (1977). Hierarchical structure in perceptual representation. Cognitive Psychology, 9, 441-474.

Palmer, S. E. (1978). Structural aspects of visual similarity. Memory \& Cognition, 6, 91-97.

Palmeri, T. J., \& Gauthier, I. (2004). Visual object understanding. Nature Neuroscience, 5, 291-303.

Piccini, C., Lauro-Grotta, R., Michela Del Viva, M., \& Burr, D. (2003). Agnosia for global patterns: When the cross-talk between grouping and visual selective attention fails. Cognitive Neuropsychology, 20, 3-25.

Ratcliff, G., \& Newcombe, F. A. (1982). Object recognition: Some deductions from the clinical evidence. In A. W. Ellis (Ed.), Normality and pathology in cognitive functions (pp. 147-171). New York: Academic Press.

Rentschler, I., Juttner, M., Osman, E., Muller, A., \& Caelli, T. (2004). Development of configural 3D object recognition. Behavioural Brain Research, 149, 107-111.

Ricci, R., Vaishnavi, S., \& Chatterjee, A. (1999). A deficit of intermediate vision: Experimental observations and theoretical implications. Neurocase, $5,1-12$.

Riddoch, M. J., \& Humphreys, G. W. (1987). A case of integrative visual agnosia. Brain, 110, 1431-1462. 
Riddoch, M. J., \& Humphreys, G. W. (1993). Birmingham Object Recognition Battery. Hove, England: Erlbaum.

Riddoch, M. J., Humphreys, G. W., Blott, W., Hardy, E., \& Smith, A. D. (2003). Visual and spatial short-term memory in integrative agnosia. Cognitive Neuropsychology, 2, 641-671.

Saiki, J., \& Hummel, J. E. (1998a). Connectedness and part-relation integration in shape category learning. Memory \& Cognition, 26, 11381156.

Saiki, J., \& Hummel, J. E. (1998b). Connectedness and the integration of parts with relations in shape perception. Journal of Experimental Psychology: Human Perception and Performance, 24, 227-251.

Saumier, D., Arguin, M., Lefebvre, C., \& Lassonde, M. (2002). Visual object agnosia as a problem in integrating parts and part relations. Brain Cognition, 48, 531-537.

Snodgrass, S. G., \& Vanderwart, M. A. (1980). A standardised set of 260 pictures: Norms for name agreement, image agreement, familiarity, and visual complexity. Journal of Experimental Psychology: Learning, Memory, and Cognition, 6, 174-215.

Tanaka, J. W., \& Farah, M. J. (1993). Parts and wholes in face recognition. Quarterly Journal of Experimental Psychology: Human Experimental Psychology, 46(A), 225-245.

Tarr, M. J., Bülthoff, H. H., Zabinski, M., \& Blanz, V. (1997). To what extent do unique parts influence recognition across changes in viewpoint? Psychological Science, 8, 282-289.
Tarr, M. J., \& Pinker, S. (1989). Mental rotation and orientation dependence in shape recognition. Cognitive Psychology, 21, 233-282.

Thaiss, L., \& de-Bleser, R. (1992). Visual agnosia: A case of reduced attentional "spotlight"? Cortex, 28, 601-621.

Turnbull, O. H. (1997). A double dissociation between knowledge of object identity and object orientation. Neuropsychologia, 35, 567-570.

Turnbull, O. H., Laws, K. R., \& McCarthy, R. A. (1995). Object recognition without knowledge of object orientation. Cortex, 31, 387-395.

Wapner, W., Judd, T., \& Gardner, H. (1978). Visual agnosia in an artist. Cortex, 14, 343-364.

Warrington, E. K. (1982). Neuropsychological studies of object recognition. Proceedings of the Royal Society of London, Series B, 298, 15-33.

Warrington, E. K., \& James, M. (1988). Visual apperceptive agnosia: A clinico-anatomical study of three cases. Cortex, 24, 13-32.

Warrington, E. K., \& James, M. (1991). The Visual Objects and Space Perception Battery. Suffolk, England: Thames Valley Test Company.

Weisstein, N., \& Harris, C. S. (1974, November 22). Visual detection of line segments: An object superiority effect. Science, 186, 752-754.

Wheeler, D. D. (1970). Processes in word recognition. Cognitive Psychology, 1, 59-85.

Received July 20, 2005

Revision received February 9, 2006

Accepted February 14, 2006 